\title{
9 Daten - Führt der Weg über Blockchain zu Big Data?
}

\author{
Alois G. Steidel
}

Blockchain ist weder Technologie noch Produkt, sondern eine Reise und ein Prozess, der gerade erst beginnt und bereits die Services der Banken und Finanzdienstleister auf den Kopf stellt. Blockchain schafft Konsens und Vertrauen zwischen Akteuren, die sich meist überhaupt nicht kennen. Verbraucher oder Unternehmen können Werte, beispielsweise Geld, Eigentum, Services oder Verträge, sicher darüber austauschen - ohne dass eine zentrale Vermittlungsinstanz für die Authentizität der Tauschwaren geradesteht. Das beschleunigt die gesamte Abwicklung enorm und könnte den Weg zu einer völlig neuen Generation transaktionaler Anwendungen ebnen.

\section{Was ist Blockchain?}

Unter einer Blockchain (auch Block Chain, englisch für Blockkette) wird eine Datenbank verstanden, deren Integrität (Sicherung gegen nachträgliche Manipulation) durch Speicherung des Hashwertes des vorangehenden Datensatzes im jeweils nachfolgenden gesichert ist (https://de.wikipedia.org/wiki/Blockchain).

Allgemeiner gesprochen ist Blockchain eine dezentrale Datenbank zur digitalen Organisation von Eigentumsrechten. Das Besondere an diesem Prinzip ist der aus dem Netzwerk hergestellte Konsensmechanismus, der jeden einzelnen Datenbankeintrag authentifiziert. Durch die Dezentralität lässt sich hier auf Mittelsmänner oder Institutionen verzichten. Damit bietet die Blockchain einen neuen Ansatz, Transaktionen auch in der digitalen Welt sicher abzubilden und bisherige Technologien zu verdrängen. 
Technisch betrachtet ist eine Blockchain eine Datenbank, die als Kopie verteilt auf vielen Rechnern liegt. Diese Rechner bilden zusammen ein virtuelles Netzwerk. Dies impliziert, dass jeder Teilnehmer des Netzwerks die Informationen in der Datenbank lesen kann. Änderungen am Stand der Daten werden transaktional durchgeführt, also entweder komplett oder gar nicht, denn im Fehlerfall werden keine Änderungen festgeschrieben.

\section{Derzeitige Einsatzszenarien}

Eine gute Übersicht darüber, welche Themenfelder bereits besetzt wurden und was es in den USA schon alles an Umsetzungen gibt, kann man unter https://distributed. $\mathrm{com} /$ finden. Die wichtigste Voraussetzung ist die Plattform, auf der sich die Teilnehmer anmelden und zusammenarbeiten können. Diese Distributed Ledger Technology (DLT) (wörtlich „verteiltes Kontobuch“) setzt den Datenaustauschstandard fest. Einer der Anbieter für solch eine Plattform ist die amerikanische Firma Hyperledger (https://www.hyperledger.org/; https://en.wikipedia.org/wiki/Distributed_ ledger).

\section{Banken und Finanzdienstleister}

Am meisten bekannt ist die Blockchain-Technik derzeit unter dem Szenario „Bitcoin“. Eine digitale Währung, deren Sicherheit auf einem verteilten System und Mechanismen der Kryptografie basiert. Deshalb kommt sie ohne Bank oder eine zentrale Stelle aus. Es geht darum, dass man Vertrauen automatisiert. Es wird die zentrale Instanz der Banken durch eine dezentrale algorithmische Instanz abgebildet. Bitcoins lassen sich weder pfänden noch anderweitig regulieren. Innerhalb der Transaktionen wird genau vermerkt, wer wie viel Geld an wen versendet, sodass zwischen den Nutzern eine Vertrauensbasis entstehen kann (https://de.wikipedia.org/wiki/ Bitcoin). Über eindeutige, anonyme Schlüssel ist für jeden Teilnehmer im Netzwerk jederzeit und aktuell ersichtlich, wie viel Eigentum ein anderer Teilnehmer wirklich hat und jedes Eigentum kann auch nur einmalig übermittelt werden. So kann keine Blockchain, z.B. Währungen oder Eigentumsrechte, mehrfach vergeben werden und ein Eigentum auch nicht vorgetäuscht werden.

\section{HealthCare}

Im HealthCare-Bereich werden bereits unterschiedliche Plattformen mit diversen Anwendungsszenarien bereitgestellt. Diese Plattformen dienen aktuell häufig noch der Vernetzung von Early Adopters und dienen als Informationsquellen. Interessante Beispiele auch für das Gesundheitswesen liefern bspw. Distributed (https://distributed.com/) oder Blockchain Healthcare Review (http://blockchainhealthcarereview. com/). Vorreiter im Bereich Blockchain für das Gesundheitswesen im amerikanischen Markt ist derzeit die Firma Gem (https://gem.co/health), die man weiter beobachten sollte. Ein interessantes Projekt ist auch „BloodChain“, welches es ermöglicht, Blutspenden mittels neuer Technologien wie QR-Codes oder auch Blockchain nachzuverfolgen. 


\section{Szenario für Europa}

Eine große Hürde mit Blick auf die globalen Cesundheitssysteme ist, einen Austausch medizinischer Daten zwischen mehreren Akteuren für unterschiedliche Zwecke zu ermöglichen und dennoch die Datenintegrität und den Schutz der Patientendaten zu sichern.

Traditionell, erfolgt die Interoperabilität von medizinischen Daten zwischen den Institutionen anhand von zwei Modellen: Push und Pull, von denen jedes seine Stärken und Schwächen hat. Blockchain bietet nun ein drittes Modell, mit dem Potenzial, einen sicheren Austausch der Krankenakten zwischen den Akteuren zu ermöglichen.

Push ist die Idee, das die medizinische Information von einem Anbieter zum anderen gesendet wird. Um eine verschlüsselte Übertragung zwischen Sender (z.B. ein Krankenhaus) und Empfänger (z.B. Ihrem Hausarzt) zu gewährleisten, bedarf es jedoch einer guten technologischen Infrastruktur, dem Vorhandensein eines elektronischen Dienstleister-Verzeichnisses für die Gemeinschaft und einer Reihe von rechtlichen Vereinbarungen. Push ist eine Übertragung zwischen zwei Parteien und keine andere Partei hat Zugriff auf die Transaktion. Wenn Sie am Ende die Information an eine weitere Partei übertragen möchten, hat diese Partei möglicherweise keinen Zugang zu den Daten. Es gibt keine Garantie für die Integrität der Daten von der Datengenerierung bis hin zur Datennutzung.

Pull beschreibt den Prozess der Abfrage von Informationen eines Anbieters durch einen anderen Anbieter, beispielsweise eines Kardiologen von einem Hausarzt. Wie bei Push, ist aber die Zustimmung und Berechtigungsvergabe notwendig. Es müssen in diesem Fall von jeder Partei, die an diesem Verfahren teilnehmen will, explizit Schnittstellen und Übertragungsverfahren definiert werden.

Diese beiden Ansätze funktionieren technisch, aber die Richtlinien, die sie umgeben, unterliegen institutioneller Variation, lokaler Praxis, staatlichen Gesetzen und der Strenge der nationalen Datenschutzrechte. Und wir gehen da auch mal wieder einen deutschen Weg. Über das Verfahren der Gematik (https://www.gematik.de). Das bedeutet, es funktioniert nur in Deutschland. Was passiert jedoch, wenn ich als Patient im Ausland bin? Als Unternehmer muss immer im europäischen Kontext oder sogar darüber hinaus gedacht werden. Die Skalierbarkeit von Investitionen hat hier Priorität.

Blockchain ist ein neuartiges Konstrukt und bietet ein universelles Set von Werkzeugen für die kryptografische Gewährleistung von Datenintegrität, standardisierter Prüfung und für den Zugriff auf formalisierte „Verträge“. Durch den Proof-of-Stake-Mechanismus (wörtlich „Beteiligungsnachweis“), bei dem jeder Transaktionsteilnehmer nachweisen muss, dass er über eine spezielle Berechtigung zur Teilnahme an dem System verfügt, ist dies möglich. Jeder Teilnehmer erhält die Authentifizierung, dass er an dieser Distributed Ledger Technology (DLT) teilhaben darf. Damit könnte es ohne Rücksicht auf staatliche Grenzen, über diese hinaus genutzt werden.

Blockchain wurde ursprünglich als DLT für finanzielle Transaktionen konzipiert. Jedes Finanzinstitut erstellt eine kryptografisch gesicherte Liste aller Einzahlungen und Auszahlungen. Blockchain nutzt öffentliche kryptografische Techniken um eine unveränderliche Kette von mit Zeitstempel versehenen Inhalten zu erstellen, an die nur weiter angehängt aber keine Information gelöscht oder verändert werden kann. 
Kopien der individuellen Blockchain sind auf vielen teilnehmenden Knoten im Netzwerk verteilt, was sie vor Manipulationen schützt. Denn die Informationen einer Blockchain müssten in fast allen Kopien gleichzeitig verändert werden, um das Original zu verändern. Über die Verteilung ist dies aber praktisch unmöglich für Hacker durchzuführen. So lange die Mehrheit von Kopien übereinstimmen, ist die OriginalBlockchain legitim.

Heute versuchen die Menschen, manuell medizinische Daten zwischen Kliniken, Krankenhäusern, Laboren, Apotheken und Versicherungsgesellschaften in Einklang zu bringen. Dies bietet viele Möglichkeiten für Fehler, denn es gibt keine Liste aller Orte, wo die Daten gefunden werden können oder Informationen über die Reihenfolge, in der sie eingegeben wurden. Theoretisch könnte eine Liste jedes jemals verschriebenen Medikamentes angefertigt werden, unklar ist jedoch, ob der Patient es letztendlich wirklich eingenommen hat.

Ein mögliches Anwendungsgebiet der Blockchain könnte eine Patientenzentrierung sein, bei der der Patient Verwalter seiner eigenen Daten ist (Eric Topol 2015). Alle Daten des Patienten sind in seiner Hand und werden zentral verwaltet. Denn erfasst werden die Daten heutzutage ja nicht mehr nur in Laboren, bei Ärzten oder Krankenhäusern, sondern auch durch unsere Fitnesstracker, Wearables oder über unsere Smartphones.

Stellen Sie sich weiter vor, dass zu diesen Datensätzen zusätzliche Informationen über Medikamente, Probleme, Allergien und ähnliches in ein gemeinschaftsweites vertrauenswürdiges DLT geschickt würden, sodass Ergänzungen und Subtraktionen in der Patientenakte gut verstanden und prüfbar organisationsübergreifend nachvollziehbar wären. Statt nur der Daten aus einer einzigen Datenbank, könnte die Patientenakte Daten aus jeder Datenbank, die im DLT autorisiert und durch den Patienten freigegeben ist, anzeigen. Das Endergebnis wäre eine perfekt abgestimmte gemeinschaftsweite Kette von Informationen über den einzelnen Fall, mit garantierter Integrität vom Beginn der Datengenerierung bis hin zur Nutzung, ohne manuellen Eingriff. Der Patient kann jederzeit selbst bestimmen, wer seine Daten sehen kann und zu welchem Zweck sie verwendet werden dürfen.

Blockchain für das Gesundheitswesen ist noch sehr früh im Lebenszyklus, aber hat bereits das Potenzial, einen sicheren Datenaustausch in unbürokratischer Weise zu standardisieren.

\section{Wie kann es weitergehen?}

Die Infrastruktur bietet die Möglichkeit, Serviceangebote für den Patienten zu erstellen. Die Daten des Patienten stellen ja einen Wert dar. Der Patient könnte entscheiden, ob er seine Daten verkauft, z.B. für eine Pharmastudie, oder ob er sie als Datenspende für Forschungszwecke bei einer Krebstherapie bereitstellt.

Analog zu einem Geo-Informationssystem mit mehreren Schichten sollte für jedes Individuum eine eigene Datenschicht, z.B. für das Genom, den Metabolismus oder den Proteinstatus erstellt sein. Für jede dieser Ebene kann der Dateneigentümer (Patient) dann eine Freigabe erteilen oder entziehen. Damit könnte man wirklich BigData-Anwendungen über Patientendaten laufen lassen, da man - die Freigabe des Patienten vorausgesetzt - umfangreiche Datengrundlagen zu Patientencluster vor- 
liegen hat und keine mühsame Datenextraktion vornehmen und die Daten in einer einheitlichen Form vorliegen.

Durch die parallelen Welten von Klinikdaten, Medical Devices, Wearables und den daraus entstehenden intelligenten Kombinationen kann ein zusätzlicher Nutzen für den Patienten bereitgestellt werden. Verarbeitet und analysiert werden diese Daten nicht mehr von menschlichen Ärzten, sondern von Algorithmen, die sich tief durch solche Datenbestände fräsen können. In Zukunft könnte das Smartphone dann die eigenen Gesundheitsdaten auswerten und per Algorithmus eine Diagnose stellen. Patienten greifen somit auf die neuesten Forschungsergebnisse zu und kommen bestens vorbereitet mit einer Vielzahl von Daten aus verschiedenen Quellen in ein Arztgespräch. Die bisherigen Konzepte zur Datenerhebung (Anamnese) und kurzen Informationsvermittlung am Ende eines Arztbesuches funktionieren in Zukunft nicht mehr. Dies sind keine abstrakten Spinnereien, sondern schon in wenigen Jahren umsetzbare und realistische Konzepte. Die Blockchain-Technologie könnte dabei einen entscheidenden Impuls geben.

\section{Literatur}

Topol E (2015) The Patient Will See You Now: The Future of Medicine is in Your Hands. Basic Books, New York, NY 